\title{
Thème 2
}

\section{Efforts de la mer sur les structures}

Rapport général

par

\author{
G. Susbielles
}

Institut français du pétrole

Rueil-Malmaison

La détermination des efforts exercés par les vagues sur les ouvrages est l'étape la plus importante de leur processus de conception, celle où souvent l'ingénieur doit intervenir pour effectuer des choix et des approximations; choix des moyens d'étude à mettre en œuvre, le modèle physique, le modèle mathématique ou les deux, approximations car dans l'un et l'autre cas la représentation de la réalité n'est pas stricte.

Pour introduire les six communications qui sont inscrites au programme de ce thème, le rapporteur a choisi de vous présenter une synthèse des progrès réalisés dans les domaines suivants : modèles mathématiques (pour grands et petits corps) mouvements des corps flottants, mesures sur les ouvrages, moyens d'essais et pour commencer, mesures de l'environnement (houle, vent, courant).

Ce qui suit est largement inspiré de mes préoccupations actuelles à l'IFP et des comptes rendus du Congrès de l'ISSC qui s'est tenu à Gênes du 23 au 27 septembre dernier.

De mon mieux, j'essayerai de conserver un juste équilibre entre les préoccupations des spécialistes du génie côtier et ceux du génie maritime pétrolier, afin de ne pas trop trahir mes origines.

Suivons donc un ordre logique.

\section{Connaissance de l'environnement marin}

Aucun thème ne traitant ce sujet, il ne me semble pas possible de le passer sous silence.

Vagues, vents et courants n'ont pas la même importance en génie maritime pétrolier et en génie côtier. Si les vagues sont importantes pour tous, les courants doivent être plus étudiés en génie côtier qu'en génie maritime pétrolier, où les opérations se déroulent en général assez loin des côtes. De même, les vents ont-ils plus d'intérêt pour l'ingénieur du génie maritime pétrolier qu'en génie côtier?

Les valeurs des grandes vagues qui caractérisent l'environnement marin sont obtenues à partir de campagnes de mesures de durée plus ou moins longue ou à partir des observations effectuées à bord des navires.
Les champs de vagues peuvent être obtenus à partir des champs de vents, en utilisant des modèles numériques: c'est la méthode de reconstitution du champ plus connue sous son appellation britannique de "hindcasting ".

Les besoins des utilisateurs, suivant qu'il s'agit de conception d'ouvrages (pétroliers ou de génie côtier) ou d'opérations en mer, sont très différents : ils vont jusqu'au spectre directionnel des vagues.

Il ne faudrait pas croire non plus que toutes les régions sont aussi bien connues que la Mer du Nord, il en existe encore beaucoup où il n'y a pas de données disponibles.

Bien que la qualité des observations faites à partir des navires reste discutable, ce qui apparaît pour l'obtention de statistiques et de valeurs extrêmes, elles ont l'avantage 
de couvrir de larges zones du globe.

Le nombre de mesures de vagues a considérablement augmenté pendant ces dernières années mais rares sont encore les sites où l'on peut disposer des résultats de mesures sur des durées suffisamment longues pour se livrer à une analyse statistique des vagues (il faut 2 à 5 ans pour des statistiques non extrêmes, 10 à 20 ans au moins, pour les valeurs extrêmes). Des questions se posent toujours quant à la fiabilité des systèmes de mesures sur de longues durées ( 5 ans).

Avant d'utiliser des données pour des extrapolations à d'autres sites que le site de mesure, il faut tenir compte de l'irrégularité du fond et du fetch.

Souvent les données les plus intéressantes sont la propriété d'organismes gouvernementaux, d'associations ou de sociétés privées, ce qui en restreint l'accès. La mesure des caractéristiques de vagues à partir des satellites est très prometteuse mais elle ne commencera qu'à la fin de cette décennie.

Les modèles de reconstitution des champs de vagues (wave hindcast) sont très largement utilisés pour des durées de prévision de 3 à 6 heures, mais aussi pour la recherche des tempêtes extrêmes survenant sur un intervalle de temps donné. La qualité de la prévision dépend de la qualité du modèle et des données de vent.

Il existe deux types de modèle, l'un dans lequel l'énergie des vagues provient, pour chaque composante (en fréquence et en direction), de l'atmosphère, l'autre dans lequel le vent apporte son énergie à une partie du spectre, cette énergie étant ensuite répartie par interactions non-linéaires entre vagues. Ils ont été utilisés pour plusieurs océans ou pour des parties d'océans.

Dans l'analyse statistique et la prédiction des houles aléatoires, des progrès assez sensibles ont été réalisés, surtout pour la prédiction.

Des formulations ont été mises au point qui permettent de lier hauteur de vague et période. La fonction de distribution de probabilité conjointe joue un rôle important dans l'étude des houles déferlantes et des groupes de vagues.

En ce qui concerne les grandes vagues en profondeur d'eau finie (faible), des fonctions densité de probabilité ont été proposées pour les processus aléatoires non-gaussiens.

Des études récentes ont montré que le $4^{c}$ moment du spectre avait une influence sur le déferlement. Une méthode a été mise au point pour la prédiction statistique des pressions d'impact des vagues déferlantes sur un cylindre vertical.

En profondeur d'eau intermédiaire, diffraction et radiation sont essentiellement des processus linéaires, alors que l'atténuation des vagues et la variation de hauteur avec la profondeur sont des processus quasi-linéaires.

Tous ces phénomènes sont prédits et analysés à partir de fonctions de transfert appliquées au spectre directionnel. Toutefois, les études possibles sont en nombre limité à cause de la rareté des données disponibles.

Plusieurs formulations ont été proposées pour la distribution des vagues non-linéaires. On cherche à relier l'écart par rapport à une distribution gaussienne à d'autres paramètres (hauteur des crêtes et profondeur des creux).

Pour les distributions de hauteurs de vagues en eau peu profonde, des modifications apparaissent par rapport à une distribution de Rayleigh, modifications qui dépendent de la non-linéarité.

De même, en faible profondeur, la densité spectrale d'énergie n'est plus une fonction de $\mathrm{f}^{-5}$ mais de $\mathrm{f}^{-4}$ et $\mathrm{f}^{-3}$.

Enfin, pour décrire le déferlement, la distribution des vagues en eau peu profonde est considérée en fonction de la profondeur d'eau locale, de la pente du fond et des caractéristiques des vagues en eau profonde.

Un certain essor mérite d'être noté dans le développement des modèles du hindcasting; plus de 20 sont actuellement disponibles, des comparaisons ont même été effectuées entre eux (voir rapport SWAMP).

En ce qui concerne les critères de conception des ouvrages, des marges de sécurité prises en compte sont d'une importance capitale pour la durée de vie et la fiabilité. Elles dépendent de la manière dont les conditions d'environnement sont transformées en cas de chargement et ensuite en contraintes.

La période de retour à adopter sera fonction de la durée du phénomène, suivant qu'il s'agit de la durée de vie totale d'un ouvrage ou d'une opération ponctuelle. (Elle peut varier de 50 ans à 1 an, nécessitant 20 ans de données ou une seule année).

Pour le vent, des mesures effectuées ont montré que pour en avoir le gradient, il était préférable d'utiliser une loi en puissance 0,125 .

Quant à la vague de projet, il est intéressant de noter qu'en Mer du Nord, on a constaté l'existence de vagues isolées associées à des interactions non-linéaires entre vagues, ayant une tendance à venir d'une direction s'écartant de 30 à $50^{\circ}$ de la direction principale des vagues.

Sur le champ d'Ekofisk, une vague a atteint une plate-forme de $20 \mathrm{~m}$ au-dessus du niveau de l'eau au repos alors que la hauteur de la vague de projet n'est que de 20 à $22 \mathrm{~m}$ sur ce site.

Dans la zone danoise, sur le champ de Gorm, durant une tempête, une vague de $17 \mathrm{~m}$ a été mesurée alors que la hauteur significative n'est que de $6,5 \mathrm{~m}$. Pour ces vagues, la hauteur de crête est de $75 \%$ de la hauteur totale de la vague.

Il est possible que les bouées de mesures n'évaluent pas correctement ces grandes vagues tridimensionnelles, ce qui entrainerait une sous-évaluation de la vague de projet.

L'attention des ingénieurs et des océanographes est maintenant concentrée sur la simultanéité (ou non) des événements extrêmes (vagues, vents et courants).

La communication présentée par SNEAP ( $\mathbf{M}^{\text {tle }}$ Lebas) concerne l'action de la vague de projet sur un ouvrage et les actions des vagues de moindre hauteur mais de période différente, aboutissant à la conclusion que sur une structure fixe force horizontale et moment de renversement n'atteignent pas simultanément leurs valeurs extrêmes.

\section{Les modèles mathématiques}

L'outil mathématique trouve sa place aussi bien dans les études du génie côtier que dans les études de génie maritime pétrolier mais les problèmes à résoudre sont très différents. En effet, en offshore, l'estimation des forces sur les structures est essentielle alors qu'en génie côtier, d'autres problèmes se posent comme le transport de sédiments. 


\section{En génie côtier}

Les modèles mathématiques concernent

- la houle et sa diffraction avec détermination de l'impact sur les courants côtiers,

- les courants au voisinage des côtes,

- les courants de marée,

- l'agitation portuaire,

- les transports de sédiments avec modèles aux différen-

ces finies puis maintenant aux éléments finis.

Des programmes plus complexes peuvent être établis faisant intervenir la turbulence, les écoulements stratifiés, les courants induits par les vagues, modèles qui peuvent être tridimensionnels.

\section{En génie maritime pétrolier}

Les problèmes sont plus simples à résoudre car la profondeur d'eau est suffisamment grande. Des approximations de $1^{\text {er }}$ ordre peuvent suffire.

Deux catégories de cas se présentent, celle des grands corps et celle des petits corps.

Forces exercées sur les grands corps: le calcul des efforts exercés par les vagues sur les corps de grande dimension s'effectue en tenant compte de la déformation de la vague par le corps (diffraction) à partir d'une théorie de $1^{\text {er }}$ ordre.

Si les principes de base de tels calculs ont été exposés dès 1949, ils ont été mis en œuvre, en France, quelques années plus tard par les ingénieurs du LNH (EDF, Chatou). Le relai a été pris par l'IFP en 1970 puis par l'ENSTA et l'ENSM de Nantes.

Au départ, la méthode consistait à introduire des singularités sur la frontière du corps à représenter (sources et puits). Bien qu'elle soit toujours utilisée, l'augmentation de la capacité de traitement des ordinateurs a permis l'introduction des éléments finis (discrétisation du fluide).

Dans un désir de simplifications et d'économie de durée de calcul, d'autres approches sont apparues avec l'introduction, non plus de puits et de sources, mais de doublets.

Plusieurs laboratoires, sociétés de classification ou organismes de recherche ont, sur ces bases, développé leurs propres codes de calcul.

Les modèles de diffraction par les grands corps sont basés sur des hypothèses simplificatrices : la surface libre est assimilée à un plan horizontal choisi comme origine des pressions, la surface mouillée du corps est supposée fixe et définie par la surface correspondante en l'absence de tout mouvement, la houle est du $1^{\text {er }}$ ordre.

C'est pourquoi des travaux actuellement poursuivis sont orientés vers des développements au $2^{\mathrm{e}}$ ordre, ce qui met en jeu un très grand volume de calculs.

Le problème est non-linéaire et lorsque le corps flottant est en mouvement, les approches ci-dessus consistent à décrire et le mouvement du corps et le mouvement du fluide, ce qui oblige à discrétiser le fluide, au moins au voisinage du corps.

Peut-être ces nouveaux modèles seront-ils applicables en faible profondeur?

Mouvements des corps flottants: le comportement des structures ancrées est caractérisé par les composantes des mouvements et des tensions d'ancrage en fonction de la fréquence (ou de la période) des vagues. Dans le cas des mouvements horizontaux, il faut y ajouter les composantes de grande amplitude à basse fréquence.

Ces mouvements sont estimés à partir de la théorie du potentiel avec des modèles bi-dimensionnels ou tridimensionnels.

Dans ces dernières années, de nombreuses études ont été orientées vers le calcul des forces de dérive ( $2^{\mathrm{e}}$ ordre) dues aux vagues, des mouvements résultants et des tensions d'ancrage correspondantes.

Les principaux travaux portent sur le comportement des tankers et des plateformes semi-submersibles.

Il n'empêche que l'amortissement des mouvements de basse fréquence n'est pas encore correctement déterminé. Les coefficients correspondants sont faibles et comme ils sont calculés à partir de la théorie du potentiel, ils sont sous-estimés car les effets dus à la viscosité ne sont pas pris en compte. Il semble que la présence des vagues augmente la valeur des coefficients d'amortissement. Des tentatives ont été effectuées pour établir des statistiques de forces de dérive.

Bien que de nombreux essais en bassin aient été effectués, le manque de données se fait encore sentir et empêche de confirmer la validité des hypothèses présidant aux équations de base.

En génie côtier, se pose aussi le problème du comportement des navires à quai. Il se résout à partir de la théorie du potentiel.

La communication présentée par I.F.P. fait le point sur les travaux entrepris dans ce domaine à l'I.F.P.

Après la mise au point de plusieurs modèles de diffraction-radiation le cas des mouvements de basse fréquence a été traité avec création de codes maintenant très utilisés pour résoudre les problèmes posés par les compagnies pétrolières (ancrages en pleine mer).

Forces exercées sur les petits corps: L'action des vagues sur des corps de petites dimensions est un problème spécifique du génie maritime pétrolier, se posant pour les plateformes de production en acier (du type treillis-jacket), et pour les canalisations sous marines, et les liaisons souples (conduites flexibles et risers).

Les travaux de recherche sur ce sujet ont réellement commencé vers 1950, s'orientant suivant deux voies : l'une théorique pour permettre la compréhension des phénomènes, l'autre plus empirique pour donner aux ingénieurs des formules à appliquer - un outil de travail pour la conception des ouvrages.

Une très grande partie des études effectuées est centrée sur la vérification de la validité (ou la non-validité) de la formule de Morison. Le phénomène physique, le décollement des tourbillons en régime d'écoulement instationnaire est très complexe. Même à l'aide des plus puissants ordinateurs disponibles, il n'est pas possible de modéliser un écoulement turbulent dû aux vagues autour d'un cylindre vertical, d'une manière à la fois précise et réaliste.

Bien que beaucoup de progrès aient été réalisés au cours des dix dernières années grâce à des essais en canal à houle et à des expérimentations en écoulement alterné dans un tube en U par le professeur Sarpkaya, beaucoup d'aspects restent à élucider tels que : le détachement des tourbillons des corps présentant des angles aigus dans la superposition de vagues et de courants ou dans un écoulement oscillant à moyenne nulle, les distributions de 
pression sur les corps, la relation entre forces longitudinale ou transversale et les nombres de Reynolds, de Keulegan et Carpenter et la rugosité, la réponse dynamique des structures déformables, les effets d'un écoulement stratifié, le retour des tourbillons, la multidirectionalité des vagues, la cinématique des vagues, les effets d'interférence dans le cas des groupes de cylindres.

Pour les actions correspondant à de grandes valeurs du nombre de Reynolds $\left(10^{6}\right)$ - cas des ouvrages en mer des méthodes ont été élaborées basées sur des distributions de tourbillons ponctuels.

Dans les écoulements périodiques, un énorme travail reste à accomplir car, par exemple, l'évolution de la position des points de décollement de la couche limite n'est pas connue, tellement la situation est complexe (cas du cylindre vertical).

L'état de la couche limite varie au cours d'un cycle suivant que l'écoulement peut être successivement laminaire, transitionnel ou turbulent.

La formule de Morison est une solution approximative à un problème très compliqué. La liste des limitations qui lui sont attachées est très longue mais il n'empêche qu'aucune autre formule n'est venue la remplacer.

Que les expérimentations soient effectuées en mer ou en bassin (canal à houle), il y a toujours des écarts entre les forces calculées et les forces mesurées sur les obstacles (cylindre vertical ou horizontal, en général).

Certains ont même tenté d'introduire des termes complémentaires et d'autres de proposer une formulation pour le calcul des forces de portance.

Des approches tout à fait différentes ont été mises en œuvre pour apporter une aide à l'ingénieur qui conçoit et qui, non obstant les incertitudes, doit calculer des efforts et des chargements.

Les efforts dus aux vagues sur une section de cylindre (en bassin) furent mesurés pour différentes conditions de houle irrégulière et en même temps la vitesse du fluide. Des techniques propres à l'analyse spectrale non-linéaire furent alors employées pour identifier les coefficients de la formulation de Morison. Les coefficients ainsi obtenus furent introduits dans des formules donnant les efforts et les enregistrements des efforts des vagues en fonction du temps furent reconstitués à partir des vitesses mesurées. Un bon accord fut obtenu entre les forces calculées et les forces mesurées, dans la direction de propagation des vagues (les forces de portance ne sont pas calculées par cette formulation).

Cette approche permet d'éliminer la nécessité de la représentation de l'écoulement autour du cylindre et donne accès à des coefficients moyennés très utiles pour l'ingénieur.

Par contre, il reste d'autres difficultés à surmonter : par exemple, le calcul des efforts exercés par la crête des vagues sur les ouvrages. La mesure de la cinématique dans cette partie de la vague est difficile car les dispositifs de mesure sont alternativement dans l'eau et hors de l'eau. Le sujet est maintenant à l'ordre du jour.

Sur ces thèmes, il reste encore un travail énorme à effectuer pour mieux comprendre les phénomènes physiques.

La communication présentée par l'I.M.G. se rattache à la fois à l'action des écoulements sur les petits corps et à la sédimentologie. Elle devrait marquer le début d'une série d'études.

\section{Mesures sur les ouvrages en mer}

Une évolution considérable s'est manifestée dans ce domaine au cours des vingt cinq dernières années. Ce sont surtout les ouvrages pétroliers qui en ont bénéficié car il est rare que des mesures soient faites sur des structures de génie côtier.

En 1960, alors que les plateformes fixes en acier du type treillis existaient déjà, presque comme nous les connaissons aujourd'hui mais dans des profondeurs d'eau beaucoup plus faibles, les préoccupations des ingénieurs étaient orientées vers la mesure des efforts dûs aux vagues et des caractéristiques de ces vagues (cinématique).

Il n'était alors question que de mesures sur des cylindres verticaux ou des éléments de petites plateformes (expérimentation de Chevron dans le Golfe du Mexique par exemple 1954-1958, 1960-1964).

Mettre en place une plateforme entière à des fins expérimentales n'était possible qu'en faible profondeur, à cause du coût élevé de l'opération. C'est ce qui fut réalisé aux Etats-Unis avec l'expérimentation d'Exxon (OTS) qui nécessita des dépenses de l'ordre de 3 millions de dollars pour l'acquisition des données (y compris le dispositif expérimental), sans compter les frais d'interprétation.

Tout changea avec l'implantation des plateformes de production en Mer du Nord et avec le vif désir du gouvernement norvégien de garantir la sécurité des ouvrages (1973).

Les compagnies pétrolières opérant en Mer du Nord furent incitées à effectuer des mesures très complètes, ne concernant pas uniquement l'environnement et les efforts exercés par les vagues mais aussi et surtout le comportement de la structure (mesures de contraintes et de fréquences propres) et, éventuellement, de comportement des fondations. Le cas des ouvrages en béton est quelque peu différent de celui des ouvrages en acier car les actions des vagues sont plus simples à décrire, tout l'intérêt se reportant sur la fondation, dans toute son étendue sous l'embase.

L'objectif poursuivi est la vérification du processus de conception. Il ne faudrait pas oublier que des mesures sont effectuées sur les ouvrages flottants (navires, plateformes semi-submersibles). Elles sont plus difficiles à réaliser compte tenu d'un espace disponible fort réduit et du programme de travail de ces ouvrages. Cependant, de nombreux cas ont été traités concernant les forces s'exerçant sur les ancrages, les mouvements ou les forces dues au vent.

En dehors des ouvrages de grande dimension, il faut noter l'excellente (par la qualité des résultats) opération réalisée conjointement par IFP et les filiales norvégiennes de Mobil, Elf-Aquitaine et Total (avec l'aide de la CEE), consistant à étudier pendant une année, le comportement d'une arche flexible soutenue par des bouées, arche susceptible d'être un élément des systèmes de production à développer dans l'avenir.

Les progrès de l'instrumentation ont entrainé l'augmentation du nombre des campagnes de mesure, ce qui permet maintenant le suivi du vieillissement d'un ouvrage.

La communication présentée par l'IFREMER bénéficie de l'expérience acquise dans l'analyse des résultats de mesures in situ effectuées sur des plateformes, fruit d'une coopération avec Elf-Aquitaine et Elf-Aquitaine Norge 
pour l'interprétation de mesures faites sur le site de Frigg. C'est une présentation rapide qui montre le chemin parcouru en dix ans et ébauche quelques perspectives d'avenir.

\section{Les moyens d'essai}

Quels que soient les sujets abordés, aussi bien dans le cadre du génie côtier que dans celui du génie maritime pétrolier, le recours au modèle physique est presque toujours d'un grand intérêt.

En génie côtier, c'est souvent la seule possibilité d'analyse des phénomènes, là où les modèles mathématiques sont insuffisants voire inexistants.

Les ouvrages pétroliers en sont tout de même moins tributaires car il existe de nombreux cas où le modèle mathématique suffit, et d'autres cas où le modèle physique est pris en défaut et ne peut permettre une représentation correcte des phénomènes (similitude de Reynolds - pour les petits corps).

En bassin, ou en canal à houle, l'environnement et ses caractéristiques sont presque parfaitement contrôlables.

L'évolution des équipements tend à introduire une représentation de plus en plus exacte de la mer, et de son caractère tridimensionnel.
Parti de la houle régulière, on est passé au stade de la houle irrégulière avant d'en arriver à la houle aléatoire monodirectionnelle. L'évolution des moyens de calcul permet le traitement très rapide des données mesurées et le contrôle des houles incidentes.

De même que l'on a développé les moyens de mesures in situ du spectre directionnel des vagues, on sait maintenant, avec les batteurs-serpent, reproduire le caractère tridimensionnel des vagues. Ceci suppose la commande du système par un dispositif électronique approprié et l'existence de moyens de calculs adéquats.

La génération du courant reste toujours un problème difficile.

Il n'est pas utile de mettre en action ces moyens "lourds" pour toute expérimentation. Ils doivent être réservés à des cas spécifiques d'où la nécessité d'études à partir de modèles mathématiques pour faire apparaître l'importance (ou non) du facteur tridimensionnel.

Ceci met plus que jamais en évidence la complémentarité entre le modèle mathématique et le modèle physique.

L'auteur présente donc un aperçu rapide de l'état des moyens d'essai consacrés en Europe au génie océanique.

Enfin, la communication de SOGREAH montre un cas où les forces exercées par les vagues en faible profondeur sur une structure de grandes dimensions ne peuvent être connues que par l'intermédiaire du modèle physique.

\section{Conclusion}

De réels progrès ont été effectués, ces dernières années, dans tous les domaines qui viennent d'être examinés. L'évolution se poursuit et semble surtout bénéficier à la Grande Bretagne, la Norvège et la Hollande sauf sur quelques points précis, la France suit cette évolution avec un certain retard. Un effort est nécessaire pour que nous revenions au niveau des meilleurs.

A la suite de la présentation du rapport général, faite par $M$. Ozanne au nom de M. Susbielles actuellement souffrant, $M$. Ozanne prononce quelques mots d'introduction avant l'exposé sur les "Motivations et les moyens de quelques instrumentations in situ d'ouvrages en mer".

Cet exposé se rapporte aux méthodes de mesure appliquées aux ouvrages en mer instrumentés, alors que le titre de la conférence prévue, qui figure dans le programme distribué, est un peu différent "Programme d'instrumentation du tripode d'Ouessant (P.I.T.O.T.) ". Je vous dois donc un mot d'explication :
Le Service technique des phares et balises va installer prochainement au large de l'Ile d'Ouessant un très grand phare et nous avons pensé à l'IFREMER et à l'I.F.P. qu'il était intéressant de se servir de cette structure comme d'une structure test pour faire un certain nombre d'expérimentations dont les résultats soient généralisables à d'autres ouvrages en mer. Il se trouve que le programme de construction de cet ouvrage des Phares et Balises est un peu retardé; il est donc trop tôt aujourd'hui pour parler en détail du programme d'instrumentation correspondant et pour en faire le sujet d'une conférence particulière. C'est pourquoi l'exposé qui va vous être présenté traite, de manière plus large, de plusieurs exemples qui ont motivé des mesures en mer. 